\title{
A multipixel diffuse correlation spectroscopy system based on a single photon avalanche diode array
}



\begin{abstract}
${ }^{1}$ Department of Biomedical Engineering, Linköping University, Linköping, Sweden

${ }^{2}$ Dipartimento di Elettronica, Informazione e Bioingegneria, Politecnico di Milano, Milan, Italy
\end{abstract}

\section{*Correspondence}

Johannes D. Johansson, Department of Biomedical Engineering, Linköping University, 58185 Linköping, Sweden. Email: johannes.johansson@liu.se

\begin{abstract}
The autocorrelation of laser speckles from coherent near infrared light is used for noninvasive estimates of relative changes in blood perfusion in techniques such as laser Doppler flowmetry (LDF) and diffuse correlation spectroscopy (DCS). In this study, a 2D array of

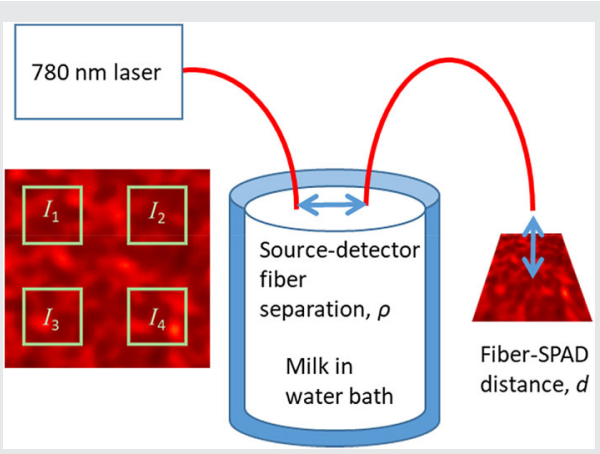
single photon avalanche diodes (SPADs) was used to combine the strengths of multiple detectors in LDF with high light sensitivity in DCS. The system was tested on milk phantoms with varying detector fiber diameter $(200$ and $600 \mu \mathrm{m})$, source-detector fiber separation (4.6-10.2 mm), fiber-SPAD distance (2.5-36.5 mm), contiguous measurement time per repetition for the autocorrelation $(1-33 \mathrm{~ms})$ and temperature $\left(15.6-46.7^{\circ} \mathrm{C}\right)$. An in vivo blood occlusion test was also performed. The multipixel approach improved signal-to-noise ratio (SNR) and, in our setup, the use of a multimode detector fiber was beneficial for SNR. In conclusion, the multipixel system works, but improvements and further studies regarding, for example, the data acquisition and optimal settings are still needed.
\end{abstract}

\section{K E Y W O R D S}

blood flow measurements, diffuse correlation spectroscopy, laser Doppler flowmetry, SPAD array

\section{1 | INTRODUCTION}

The electromagnetic nature of light allows for interference of its electric field. Coherent lasers have a very narrow spectral broadening around its wavelength and the interference from such light can form clear speckle patterns on optical detectors. These speckles from light scattered in turbid media are used for monitoring relative changes in microvascular blood flow in a number of techniques such as laser Doppler flowmetry (LDF) [1, 2], laser speckle contrast imaging [3, 4] and diffuse correlation spectroscopy (DCS) $[5,6]$. The techniques rely on the effect that moving light-scattering particles, such as red blood cells, will cause the speckle pattern to change over time. These changes are faster if there are more and faster moving particles. Near infrared light is commonly used, as tissues have a relatively low optical absorption in the range 650 to $950 \mathrm{~nm}$ [5].

LDF utilizes light collected from a short distance from the light source fiber, for example, from a detector fiber adjacent to an illuminating source fiber at a center-to-center distance of for example, $200 \mu \mathrm{m}$. As such, it mainly probes a very superficial depth in the tissue (Figure 1). As the light 


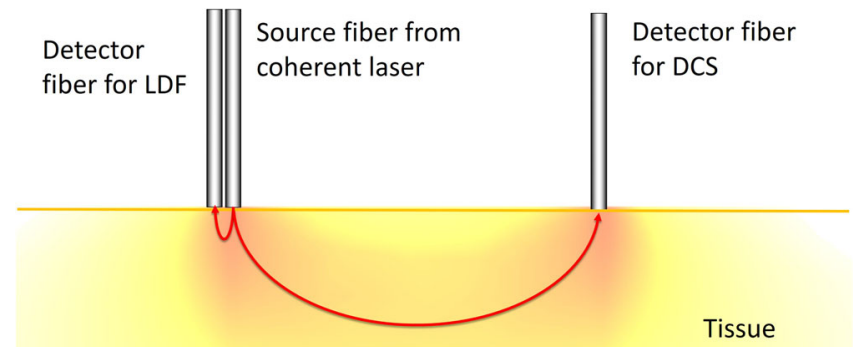

F I G URE 1 Sketch of typical fiber configurations and light trajectories for laser Doppler flowmetry (LDF) and diffuse correlation spectroscopy (DCS). In LDF, the light scattered through the tissue is collected by a detector fiber adjacent or up to a few millimeter from the source fiber while in DCS, the light is collected by a detector fiber, for example, 5 to $25 \mathrm{~mm}$ away from the source fiber. The light will travel further and deeper through the tissue before detection in the DCS case. This results in a heavily attenuated light intensity and demands a much more sensitive detector than in the LDF case. The light transport does not follow one line through the tissue but is diffuse as illustrated for the DCS case by the colored background field

is not attenuated much for such short fiber separations, relatively simple detectors without enhanced sensitivity can be used. Here, the speckles are studied by power spectral analysis of the Fourier transform of the difference in light intensity between two detector pixels. This is then used to calculate a relative perfusion estimate in the studied tissue. LDF has a sufficiently high temporal resolution (eg, $2.5 \mathrm{~ms}$ ) to allow study of blood flow changes over the cardiac cycle [7]. Due to the short penetration depth, nonsuperficial tissue can only be studied with invasive probes. LDF has been used in order to study, for example, burn wound healing [8], diabetes [9], neurosurgery [10] and skin cancer [11]. A recent development in LDF is to include optical parameters from diffuse reflectance spectroscopy in the perfusion modeling. Another is to differentiate the perfusion estimate into different speed components by the use of inverse Monte Carlo light transport modeling [12].

Despite the name, DCS is not a spectroscopic technique in the sense of using multiple wavelengths, but rather a laser speckle technique that looks at light that has been scattered across a larger distance through the tissue than is the case in LDF (Figure 1). It can, for example, utilize a detector fiber several millimeter or even centimeter from the illuminating source fiber. Here, the light travels much further through the tissue and will be much more attenuated. This puts a higher demand on the sensitivity of the detector and photon-counting avalanche detectors are thus used. The analysis in DCS is done in the time domain for a single detector on the light intensity autocorrelation, $g_{2}$, from delay times of around $1 \mu \mathrm{s}$. This autocorrelation is corresponding to the power spectrum in the Fourier domain used in LDF. It is fitted to a light diffusion model to obtain an estimate of the relative blood perfusion, which is assumed to be proportional to a Brownian diffusion term, $D_{\mathrm{b}}$. Typical analysis with hardware correlators are done at a temporal resolution of 1 second. DCS has been used, for example, to study breast cancer [13], animal model tumors [14] and cerebral blood flow in premature infants [15]. A recent development in DCS is to improve the signal quality for large source-detector separations or to overcome the limited temporal resolution. This has been done by, for example, larger detector fibers [16] or the use of multiple detector fibers and detectors to collect the light [17]. Another approach for improved temporal resolution is to use software autocorrelation [18]. Multilayer modeling has been developed to separate blood flows at different depths $[19,20]$ and coherence-maintaining laser pulsing has been used to perform simultaneous time-resolved spectroscopy and DCS. The latter technique may also provide separation of flows at different depths [21]. The new idea proposed in this study is to use a multiple pixels as in LDF for DCS for estimates of blood flow changes. A similar idea has recently been introduced for the related technique interferometric diffusing wave spectroscopy by the use of a complementary metal oxide semiconductor (CMOS) camera for cerebral blood flow measurements [22].

Single photon avalanche diodes (SPADs) are optimal detectors for combining LDF and DCS techniques. In fact, they have high sensitivity at the single photon level and they are solid-state devices, so they can be monolithically integrated with the front-end electronics in CMOS technologies and they are rugged against high photon fluxes [23].

The aim of this study is to present and characterize a new DCS system based on a multipixel SPAD array and to investigate whether it can benefit the signal-to-noise ratio (SNR) of the perfusion measurements. To the best of our knowledge, such approach has never been presented in literature.

\section{2 | MATERIALS AND METHODS}

\section{1 | System description}

\subsection{1 | SPAD array}

The detector integrated circuit (IC) employed in this work is a $5 \times 5$ pixels $2 \mathrm{D}$ array of silicon SPADs with integrated readout and quenching electronics. Each SPAD has a square active area with $50 \mu \mathrm{m}$ side length and rounded corners. The distance between the SPAD edges is $25 \mu \mathrm{m}$, giving a center-to-center distance of $75 \mu \mathrm{m}$ between adjacent pixels. Reference [24] presents a complete characterization of the performance of SPADs with the same cross-section and fabricated in the same technology. Figure 2 shows the SPAD photon detection efficiency (PDE) as function of wavelength and excess bias voltage. Peak PDE is about $45 \%$ at $480 \mathrm{~nm}$, while at $780 \mathrm{~nm}$ (the wavelength used in this experiment) 


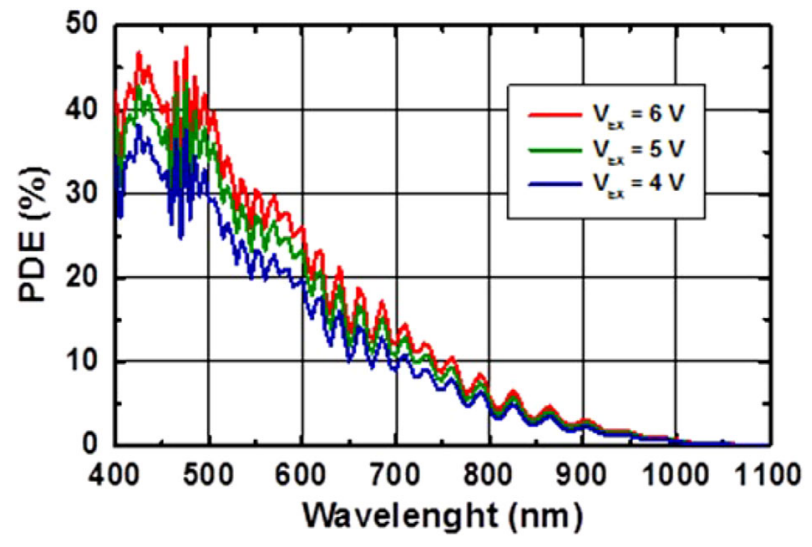

F I G URE 2 Photon detection efficiency (PDE) of the single photon avalanche diode detector as a function of wavelength measured at three different excess bias voltages. Note that the PDE at $780 \mathrm{~nm}$ (the wavelength used in this study) is $8 \%$

the PDE is $8 \%$ at $6 \mathrm{~V}$ excess bias. Typical dark count rate (DCR) for each pixel is about $100 \mathrm{cps}$, measured at $25^{\circ} \mathrm{C}$ and $6 \mathrm{~V}$ excess bias. Another issue which afflicts SPADs is the afterpulsing phenomenon, that is, the presence of carriers trapped during the avalanche that are later released, causing a spurious ignition. In application like DCS in which the autocorrelation function has to be computed, the afterpulsing probability should be as low as possible. To mitigate the impact of afterpulsing, SPAD detectors are kept quenched for a fixed time duration after each avalanche, avoiding igniting spurious avalanches due to the released carriers. In the sensor used for this experiment, the hold-off time can be configured by means of an external analog voltage with a minimum duration of $20 \mathrm{~ns}$, which corresponds to $16 \%$ afterpulsing probability. In the measurement presented in this study, the hold-off was set to $50 \mathrm{~ns}$, which corresponds to $6.5 \%$ afterpulsing probability.

Four adjacent SPADs in a square, among those available in the array, were chosen to perform the measurements based on their DCR performance as well as geometric position to minimize differences in average light intensity between the SPADs. The sensor IC provides four independent digital outputs, whose rising edges are synchronous to a photon detection. Those outputs are read by a field-programmable gate array (FPGA), which performs photon counting and integration, as well as interfacing to a PC for further data analysis. The sampling and accumulation parameters (integration window time, number of bits, hold-off time) are user-defined, allowing flexibility in the choice of sampling rate and bit depth. The sampling rate can be as fast as $1 \mathrm{MHz}$, satisfying the requirement of delay times of $1 \mu$ s for DCS applications. In the following measurement, an integration window of $1 \mu \mathrm{s}$ was used, corresponding to the maximum sampling rate. The bit depth of the photon counter implemented in the FPGA is adjustable from 4 to 16 bit. The counter bit depth should be chosen to avoid saturation of the photon counts with the incoming light signal. At the same time it should be kept as low as practically possible, since the bottleneck of the system is the data transfer from the FPGA to the PC. This is due to the "burst" nature of universal serial buffer (USB) transfers and limited memory available on the employed FPGA, which is too small to buffer data between consecutive USB transfers. The maximum "burst" size is of 32760 time points with 4 bits counter depth and 16380 time points with 8 bits. This limitation will be overcome in a future development of the system, using a more powerful FPGA.

\subsection{2 | Laser source and fibers}

Coherent continuous wave $780 \mathrm{~nm}$ near infrared laser light was generated from a commercial LDF system (Periflux 5000, Perimed AB, Sweden). The light, with a power of $0.6 \mathrm{~mW}$, was delivered to the sample and collected from it using optical fibers (source fiber and detector fiber, respectively) with a core diameter of $200 \mu \mathrm{m}$ and a numerical aperture (NA) of 0.37. A detector fiber with a core diameter of $600 \mu \mathrm{m}$ and an NA of 0.37 was also used for the light collection.

The detector fiber was mounted on a stack of SM1 tubes (Thorlabs) for variable distance to the SPAD sensor (from 2.5 to $36.5 \mathrm{~mm}$ ) and the intensity was recorded in 4 pixels (Figure 3).

\subsection{3 | Speckle area}

The size of a single speckle area, $A\left(\mu \mathrm{m}^{2}\right)$, on the detector can be estimated by [25]:

$$
A=\frac{\lambda^{2} d^{2}}{\pi r^{2}}
$$

where $\lambda$ is the wavelength of the light $(0.78 \mu \mathrm{m}), d$ is the variable distance between the detector fiber and the detector

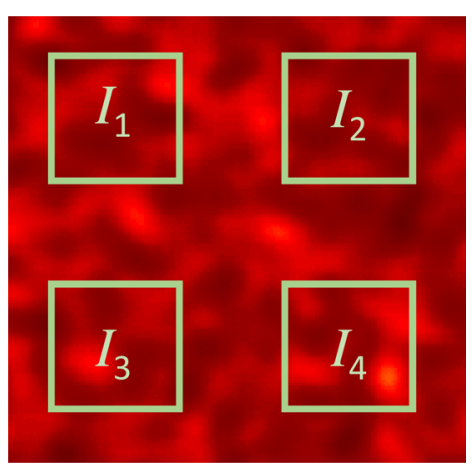

F I G URE 3 Illustration of speckle pattern on detector pixels. The speckle pattern has been obtained by spatially filtering random noise (randn and filtfilt, MatLab, Mathworks) 
(2500-36 $500 \mu \mathrm{m}$, adjustable through the stack of SM1 tubes) and $r$ the detector fiber radius (100 or $300 \mu \mathrm{m})$. Thus, the speckle area will be equal to the detector area, $A_{\mathrm{det}}$ (about $50 \times 50=2500 \mu \mathrm{m}^{2}$ ), when

$$
d=\sqrt{\frac{\pi r^{2} A_{\mathrm{det}}}{\lambda^{2}}} .
$$

For the $200 \mu \mathrm{m}$ diameter fiber, this will occur at $d=11.4 \mathrm{~mm}$ and for the $600 \mu \mathrm{m}$ fiber it will occur for $d=34.1 \mathrm{~mm}$.

\section{2 | Signal analysis}

For each measurement, 100 repetitions of light intensity in 16380 time points with a temporal resolution of $1 \mu$ s were done. Due to firmware limitations, a delay of approximately $42 \mathrm{~ms}$ was required between each repetition, giving a time of around $60 \mathrm{~ms}$ per repetition and a total time of 6 seconds for each measurement.

\subsection{1 | Electric field autocorrelation}

The normalized electric field autocorrelation for a semiinfinite geometry, $g_{1}$ (dimensionless unit, denoted by [-] in this study), was used to fit a Brownian diffusion coefficient $D_{\mathrm{b}}\left(\mathrm{cm}^{2} / \mathrm{s}\right)$ to the measurements according to $[5,6]$ :

$$
g_{1}=\frac{\left(\frac{e^{-K(\tau) r_{1}}}{r_{1}}-\frac{e^{-K(\tau) r_{2}}}{r_{2}}\right)}{\left(\frac{e^{-K(0) r_{1}}}{r_{1}}-\frac{e^{-K(0) r_{2}}}{r_{2}}\right)} .
$$

Here, $K(\tau)=\sqrt{3 \mu_{\mathrm{a}} \mu_{\mathrm{s}}^{\prime}+6 \alpha D_{\mathrm{b}} \tau \mu_{\mathrm{s}}^{\prime 2}(2 \pi n / \lambda)^{2}}$, where $\mu_{\mathrm{a}}$ is the absorption coefficient $\left(\mathrm{cm}^{-1}\right), \mu_{\mathrm{s}}^{\prime}$ is the reduced scattering coefficient $\left(\mathrm{cm}^{-1}\right), \alpha$ is the fraction of moving scatterers (-), $\tau$ is the delay time (seconds), $n$ is the index of refraction (-), $\lambda$ is the vacuum wavelength of the light $(\mathrm{cm}), r_{1}$ the distance to a virtual point source beneath the source fiber $(\mathrm{cm})$ and $r_{2}$ the distance to a virtual sink above the source fiber $(\mathrm{cm})$. These distances are related to the source-detector distance, $\rho$ $(\mathrm{cm})$, according to:

$$
\begin{gathered}
r_{1}=\sqrt{\rho^{2}+\left(\frac{1}{\mu_{\mathrm{s}}^{\prime}}\right)^{2}}, \\
r_{2}=\sqrt{\rho^{2}+\left(2 z_{\mathrm{b}}+\frac{1}{\mu_{\mathrm{s}}^{\prime}}\right)^{2}}, \\
z_{\mathrm{b}}=\frac{2}{\mu_{\mathrm{s}}^{\prime}} \frac{1+R_{\mathrm{eff}}}{3\left(1-R_{\mathrm{eff}}\right)} .
\end{gathered}
$$

where $R_{\text {eff }}$ is the effective reflection coefficient (-), going from 0 at a boundary with matched index of refraction to 1 for a perfectly reflecting boundary. The measured light intensity autocorrelation used for DCS calculations [5]

$$
g_{2}(\tau)=\frac{\langle I(t) I(t+\tau)\rangle_{t}}{\langle I(t)\rangle_{t}^{2}}
$$

can be related to $g_{1}$ by the Siegert relation:

$$
g_{2}=1+\beta\left|g_{1}\right|^{2},
$$

where $\beta(-)$ is a coefficient depending on, for example, the speckle area $A$ and the detector area $A_{\text {det }}$. This allows $g_{1}$, which is used for the theoretical fitting in Equation (3), to be obtained from $g_{2}$, which is obtained from the measurements. Delay times, $\tau$, between $10^{-6}$ and $10^{-3}$ seconds in steps corresponding to the ticks on a logarithmic scale $\left(10^{-6}\right.$ seconds steps up to $\tau=10^{-5}$ seconds, $10^{-5}$ seconds steps up to $\tau=10^{-4}$ seconds, $10^{-4}$ seconds steps up to $\tau=10^{-3}$ seconds, that is $\left[1 \times 10^{-6}, 2 \times 10^{-6}, \ldots, 9 \times 10^{-4}, 1 \times 10^{-3}\right.$ seconds]) were used to calculate $g_{2}(\tau$, pixel) in each pixel and the $g_{2}$ from the four pixels were then averaged to a single $g_{2}(\tau)$. An uneven step length for $\tau$ was chosen in order to put higher weight on short delay times. Poor fits were obtained if an even step size of $1 \mu \mathrm{s}$ was used and it also takes longer time to calculate. DCS systems generally use similar schemes with longer steps for longer delay times [26].

The SNR for $g_{2}$ was calculated as the mean $\left(g_{2}(\tau\right.$, repetition) - 1) over the 100 repetitions divided by the SD of $\left(g_{2}(\tau\right.$, repetition $\left.)-1\right)$ over the repetitions and then averaged over the used $\tau$ to a single value. Fitting of $D_{\mathrm{b}}$ and $\beta$ were done individually to the 100 repetitions using least squares fitting to $\left|g_{1}\right|^{2}$ obtained from Equation (8). Fitting was done to $\left|g_{1}\right|^{2}$ rather than $g_{1}$ in order to avoid complex numbers from noise around 0 . The initial value of $D_{\mathrm{b}}$ in the fitting was set to $10^{-8} \mathrm{~cm}^{2} / \mathrm{s}$ and the initial value of $\beta$ was taken from Equation (8) at the delay time of $1 \mu \mathrm{s}$ with $g_{1}(\tau=1 \mu \mathrm{s})$ assumed to be 1 and $g_{2}(\tau=1 \mu \mathrm{s})$ taken from the measurement. The stability of the $D_{\mathrm{b}}$ estimates was computed by calculating the mean and SD over the 100 repetitions. All calculations, including the autocorrelation in Equation (7) were performed in MatLab (Mathworks).

\section{3 | Milk phantom tests}

The system was evaluated on a beaker with $1.5 \%$ fat milk in a water bath to control its temperature. The first tests were performed at room temperature (around $20^{\circ} \mathrm{C}$ ), whereas the last test was performed at different temperatures (about from $15^{\circ} \mathrm{C}$ to $45^{\circ} \mathrm{C}$ ). For the fitting, the milk was assumed to have $\alpha=1, n=1.33, \mu_{\mathrm{a}}=0.027 \mathrm{~cm}^{-1}$, equal to that of water at 
$780 \mathrm{~nm}$ [27], and $\mu_{\mathrm{s}}^{\prime}=16 \mathrm{~cm}^{-1}$ [28]. The optical fibers were placed over the milk in a piece of highly scattering white polystyrene foam with the fiber ends in level with the foam edge. This was assumed to give the milk boundary $R_{\text {eff }}=$ 1, effectively removing the sink terms from Equation (3) by making $r_{2}$ go to infinity. Milk was used as it has similar scattering properties as living tissue. Blood flow also have a similar shape of $g_{2}$ as milk, despite not actually being Brownian in nature [5], while the motion of the scattering lipids and proteins in milk is Brownian. The diffusion approximation used for Equation (3) is generally assumed to be valid when $\mu_{\mathrm{a}} \ll \mu_{\mathrm{s}}^{\prime}$ and $\rho>3 / \mu_{\mathrm{s}}^{\prime}$ [5]. Thus, in this case, it is expected to be accurate for $\rho>0.19 \mathrm{~cm}$.

\subsection{1 | Test of impact of different setups on SNR}

Using a larger detector fiber or moving the fiber closer to the detector allows for more light to be collected but will also result in smaller speckle areas and consequently a greater number of speckles on each detector according to Equation (1). This gives higher spatial decorrelation which will reduce $\beta$. In order to investigate a suitable trade-off between these two effects for optimal SNR, the fiber-detector distance, $d$ (Figure 4), was varied in three sets with different detector fiber diameter and source-detector fiber separation, see Table 1. SNR values were also calculated for the cases of just using one detector pixel and for averaging the intensity on the detector pixels before calculating $g_{2}$.

The order of the distances, $d$, was randomized in each set. Sets 1 and 2 were recorded in 8 bits counter depth, while set 3 was recorded with 4 bits, allowing for 32760 time points per repetition, but these were cut down to 16380 in order to be comparable with Sets 1 and 2. In order to investigate the impact of the length of each contiguous repetition,

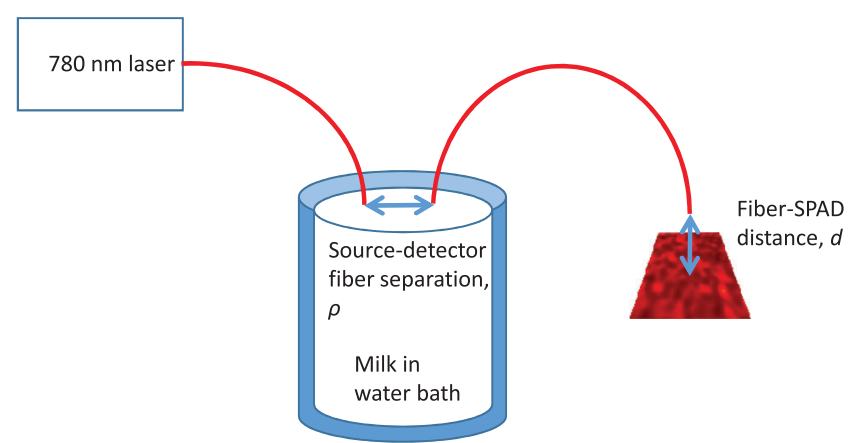

F I G U RE 4 Experimental setup with variable source-detector fiber separation and detector fiber-SPAD (single photon avalanche diode) distance. The fiber-SPAD distance was varied with a stack of SM1 tubes. The $D_{\mathrm{b}}$ of the milk was changed by varying the temperature of the water bath
TABLE 1 Variation of fiber parameters

\begin{tabular}{lclc} 
& $\begin{array}{l}\text { Fiber } \\
\text { diameter }(\boldsymbol{\mu m})\end{array}$ & $\begin{array}{l}\text { Source-detector } \\
\text { fiber separation, } \\
\boldsymbol{\rho}(\mathbf{c m})\end{array}$ & $\begin{array}{l}\text { Fiber-SPAD } \\
\text { distance, } \boldsymbol{d}, \\
\text { range }(\mathbf{m m})\end{array}$ \\
\hline Set 1 & 200 & 0.46 & $2.5-34$ \\
Set 2 & 600 & 0.51 & $5-36.5$ \\
\hline Set 3 & 600 & 1.02 & $5-36.5$ \\
\hline
\end{tabular}

SNR was also calculated for Set 3 at the full 32760 sample length and for reduced length in halving steps.

\subsection{2 | Test at different phantom temperatures}

$D_{\mathrm{b}}$ of spherical particles with a radius of $r(\mathrm{~m})$ in water is described by the Stokes-Einstein equation [29]:

$$
D_{\mathrm{b}}=\frac{k_{\mathrm{B}} T}{6 \pi \eta r}
$$

where $k_{\mathrm{B}}$ is the Boltzmann's constant $\left(1.38 \times 10^{-23} \mathrm{~J} / \mathrm{K}\right)$, $T$ is the absolute temperature $(\mathrm{K})$ and $\eta$ is the viscosity of the water (Pa s). While the temperature change for liquid water is limited from 273 up to $373 \mathrm{~K}, D_{\mathrm{b}}$ is still highly temperaturedependent due to reduced $\eta$ with increasing temperature, dropping from $1.79 \mathrm{mPa} s$ at $0.01^{\circ} \mathrm{C}$ to $0.28 \mathrm{mPa}$ at $99.6^{\circ} \mathrm{C}$ [30]. This effect was utilized to change $D_{\mathrm{b}}$ by changing the temperature of a water bath around the milk beaker. The temperature of the milk was measured with a thermocouple and DCS measurements were taken when the milk had equilibrated within $0.5^{\circ} \mathrm{C}$ of the surrounding water. Two series were made with the $200 \mu \mathrm{m}$ fiber at 2.5 and $11 \mathrm{~mm}$ fiberSPAD distance for temperatures from $15.6^{\circ} \mathrm{C}$ to $45.4^{\circ} \mathrm{C}$ and $16.4^{\circ} \mathrm{C}$ to $46.7^{\circ} \mathrm{C}$, respectively.

\section{4 | Blood occlusion test}

Finally, the system was tested in vivo on the volar forearm of a volunteering subject with Caucasian skin during blood occlusion with a pressure cuff. Written informed consent was obtained from the subject (Regional Ethical Review Board at Linköping University approval number: 2015/ 392-31). Sets of 10 measurements were made with approximately 12-second intervals during 5 minutes of baseline, 5 minutes of occlusion and 5 minutes of reperfusion. The fiber setup was a source-detector fiber separation of $5 \mathrm{~mm}$, a detector fiber of $600 \mu \mathrm{m}$ and a fiber-SPAD distance of $11 \mathrm{~mm}$. The measurements were done at 4 bits, giving 32760 time points per measurement. The optical properties of the forearm were assumed to be $\mu_{\mathrm{s}}^{\prime}=18 \mathrm{~cm}^{-1}$ and $\mu_{\mathrm{a}}=$ $0.50 \mathrm{~cm}^{-1}$ for the fittings [31]. The occlusion and reperfusion was rapid in order to avoid blood volume changes that 
can occur when the veins are occluded for a prolonged period before the arteries are occluded.

\subsection{Intralipid phantoms for longer source- detector fiber separations}

A measurement series in an Intralipid phantom was done for comparison with literature. Four percent Intralipid $(200 \mathrm{mg} / \mathrm{mL}$, Fresenius Kabi, Sweden) was mixed in distilled water for $0.8 \%$ lipid in total and $\mu_{\mathrm{s}}^{\prime}=8 \mathrm{~cm}^{-1}[32,33]$, comparable with that used by He et al [16] and Zhou et al [22]. Measurements were made for five source-detector fiber separations between 6 and $25 \mathrm{~mm}$ at $20^{\circ} \mathrm{C}$. Each measurement consisted of 100 repetitions at 4 bits. Here, the SNR was also calculated as a function of the delay time, $\tau$, for comparison with $\mathrm{He}$ et al [16].

\section{3 | RESULTS}

The impact on SNR in $g_{2}$ when changing the fiber-SPAD distance, and thus the number of speckle areas on each SPAD pixel is presented in Figure 5. More speckles per pixel results in higher light intensity, which is expected to be beneficial for the SNR, but lower $\beta$, which is expected to be detrimental to the SNR. The highest SNR values were obtained for fiber-SPAD distances corresponding to approximately 10 speckles per SPAD pixel. Average $( \pm \mathrm{SD})$ improvement from using 3 to 20 speckles compared to a matched speckle size was $32 \pm 7 \%$ for Set $1,24 \pm 6 \%$ for Set 2 and $38 \pm 7 \%$ for Set 3 (Figure 5D). In Figure 5, it is also shown that the usage of four detector pixels doubled the SNR as should be expected from averaging of four signals. Less obvious, this benefit was almost completely erased if the intensities on the pixels were averaged before calculating $g_{2}$ instead of averaging the obtained $g_{2}$. It can be noted that averaging the intensity of the pixels before calculating $g_{2}$ effectively equates using a single larger detector pixel.

Light intensity and examples of fits at the highest and lowest intensities are presented in Figure 6. It shows, as expected, that larger detector fiber, shorter source-detector fiber distance and close fiber-SPAD distance provide higher intensities. It could be seen that $g_{2}(1 \mu \mathrm{s})$ was lower than $g_{2}(2 \mu \mathrm{s})$ and the fitted curve for the highest intensities (Figure 6B). For the lowest intensities on the other hand, $g_{2}(1 \mu \mathrm{s})$ was higher than the fitted curve (Figure 6C).

Values of $\beta$ and the difference between $\beta$ and $g_{2}(1 \mu \mathrm{s})-$ 1 , which should be approximately 0 if the decorrelation at $1 \mu \mathrm{s}$ is negligible, are presented in Figure 7. It shows that $\beta$ decreases at closer fiber-SPAD distance and with larger detector fiber. Fitted $\beta$ values were slightly higher in Set 3 with longer source-detector fiber separation compared to
(A)

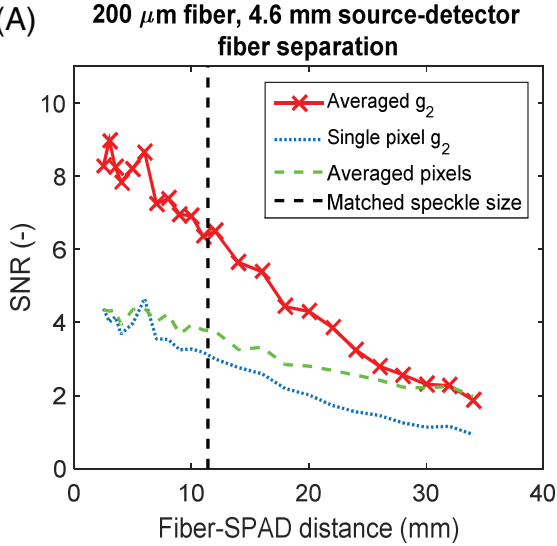

(C)

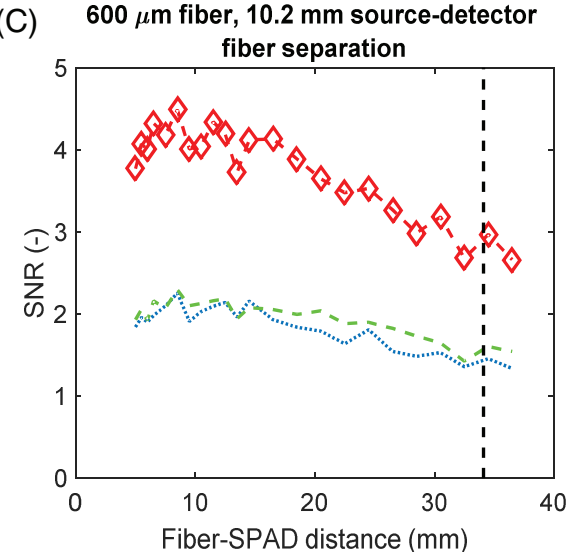

(B)

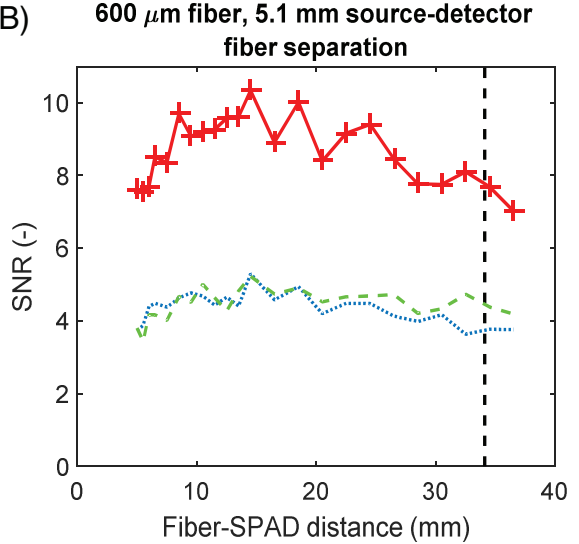

(D)

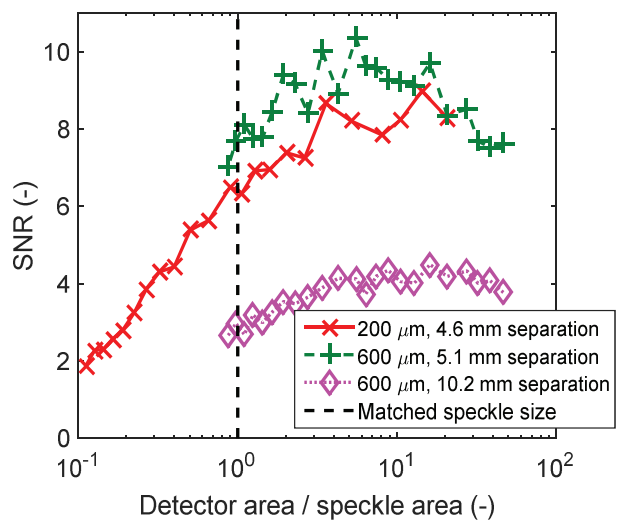

F I G U RE 5 Signal-to-noise ratio (SNR) for $60 \mathrm{~ms}$ measurements when changing fiber-detector distance for (A) Set 1, (B) Set 2 and (C) Set 3. Averaging $g_{2}$ from four pixels (red curves) approximately doubles the SNR compared to calculating $g_{2}$ from a single pixel (blue curves) or averaging the light intensity on the pixels before calculating $g_{2}$ (green curves). (D) Corresponding curves for averaged $g_{2}$ with SNR vs the ratio of detector and speckle area, that is, approximately the number of speckles per SPAD pixel. Average $( \pm \mathrm{SD})$ improvement from using 3 to 20 speckles compared to a matched speckle size was $32 \pm 7 \%$ for Set $1,24 \pm 6 \%$ for Set 2 and $38 \pm 7 \%$ for Set 3. (-) refers to dimensionless unit 
(A)

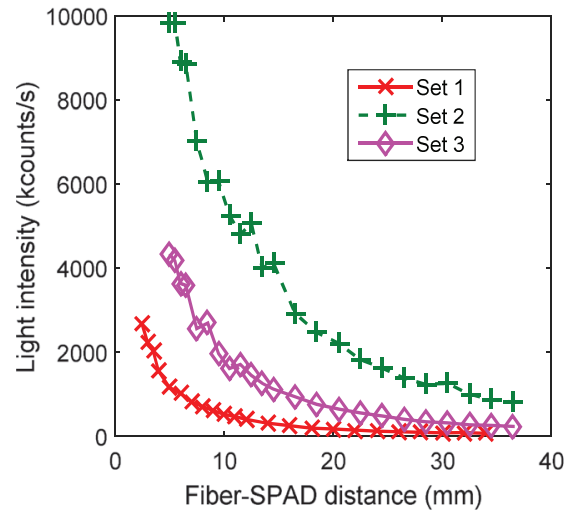

(B)

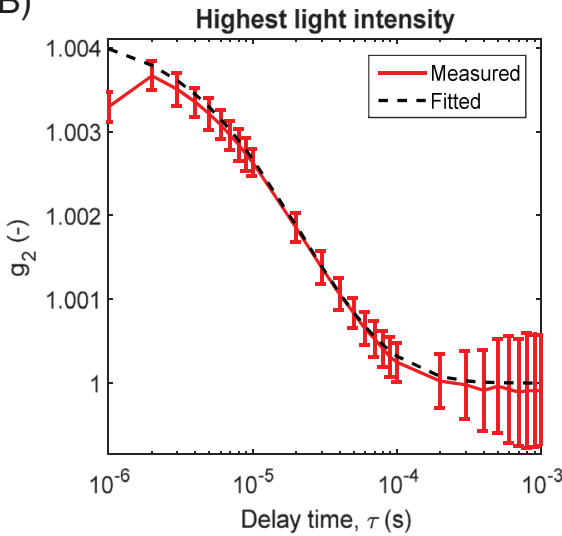

(C)

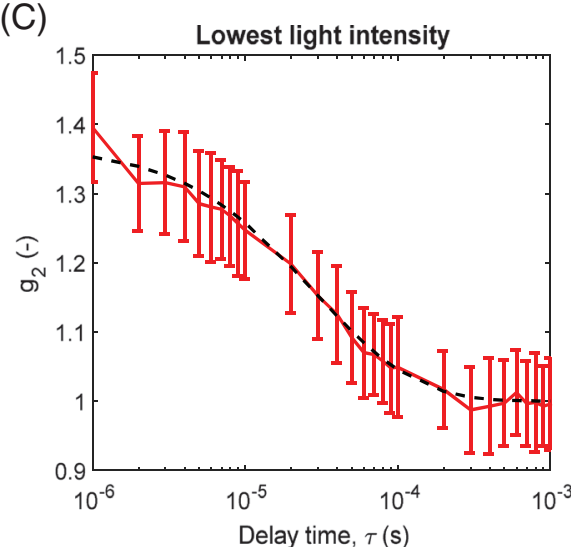

F I G URE 6 (A) Average light intensity on the detector pixels when changing fiber-detector distance. By itself, higher light intensity is expected to give higher signal-to-noise ratio (SNR). However, higher intensity from shorter fiber-SPAD distances is also associated with lower $\beta$ (Figure 7) which lowers the SNR. (B) For the highest intensities, lower values of $g_{2}(1 \mu \mathrm{s})$ than for $g_{2}(2 \mu \mathrm{s})$ could be seen, which is likely an artifact of the hold-off time eliminating photon counts in the subsequent time bin. (C) For the lowest intensities, $g_{2}(1 \mu \mathrm{s})$ tended to be higher than the fitted curve, likely due to the afterpulsing generating false photon counts in the subsequent time bin. Error bars correspond to mean and SD over 100 sets of $16.4 \mathrm{~ms}$ measurements. The fitted curves refer to the average of the sets

(A)



(B)

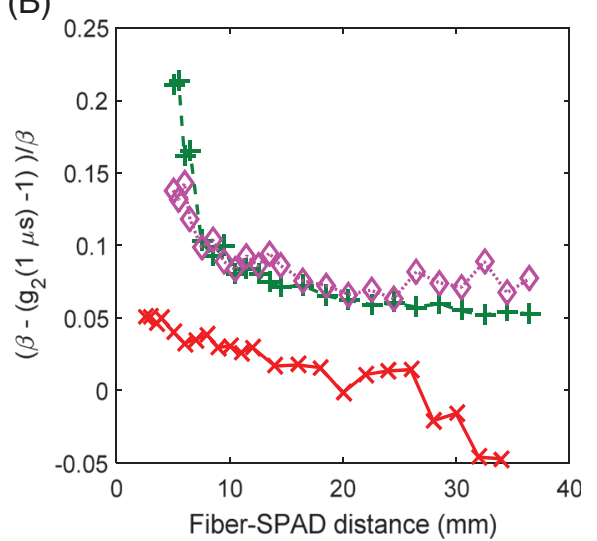

F I G URE 7 (A) Values for $\beta$ when changing fiber-detector separation. It can be noted that maximizing these values do not correspond to optimal signal-to-noise ratio as can be seen when comparing with Figure 5. (B) Average fractional difference between $\beta$ and $g_{2}(1 \mu \mathrm{s})-1$, which should be approximately 0 when the decorrelation is negligible. The reduction in $g_{2}(1 \mu \mathrm{s})$ for the highest light intensities (Figure $6 \mathrm{~B}$ ) can be seen for Sets 2 and 3 at the shortest fiber-SPAD distances. The increase of $g_{2}(1 \mu \mathrm{s})$ at the lowest light intensities (Figure 6C) can be seen at the four longest fiber-SPAD distances in Set 1 where it can be seen to be larger than the estimated $\beta$. Error bars correspond to mean and SD over 100 sets of 16.4 ms measurements

Set 2, which otherwise had the same detector fiber and fiberSPAD distance. This difference persists for $g_{2}(1 \mu \mathrm{s})-$ 1. Fitted $D_{\mathrm{b}}$ values are presented in Figure 8A. There was considerably larger variance between the sets than within them with mean \pm SD. $D_{\mathrm{b}}$ of $5.7 \times 10^{-9} \pm 0.5 \times 10^{-9} \mathrm{~cm}^{2} / \mathrm{s}$ for Set $1,6.6 \times 10^{-9} \pm 0.2 \times 10^{-9} \mathrm{~cm}^{2} / \mathrm{s}$ for Set 2 and $4.9 \times$ $10^{-9} \pm 0.3 \times 10^{-9} \mathrm{~cm}^{2} / \mathrm{s}$ for Set 3, giving an SD of $0.9 \times$ $10^{-9} \mathrm{~cm}^{2} / \mathrm{s}$ between the sets. Linear regression (MatLab) between the mean over the SD of $D_{\mathrm{b}}\left(D_{\mathrm{b}} \mathrm{SNR}\right)$ and the $g_{2}$ SNR (Figure $8 \mathrm{~B}$ ) showed a statistically significant increase in $D_{\mathrm{b}}$ SNR with increasing $g_{2}$ SNR at $P<.001$ for Sets 1 and 3 and at $P<.05$ for Set 3 . Fitted $D_{\mathrm{b}}$ values tended to increase for lower SNR. Linear regression between mean $D_{\mathrm{b}}$ and the SNR (Figure 8B) showed higher $D_{\mathrm{b}}$ for lower SNR in Set 1 at $P=.001$ and in Set 3 at $P<.02$, but no statistically significant relation in Set 2 .

The impact of the contiguous repetition length is presented in Figure 9, together with plotting of the square root of the repetition length which would be the expected improvement of signal averaging. In Figure 9C, it can be seen that reducing the contiguous repetition length, apart from reducing SNR, also can increase the estimates of $D_{\mathrm{b}}$. Low SNR may thus give overestimation of $D_{\mathrm{b}}$ and not just a greater variability in the estimates. 

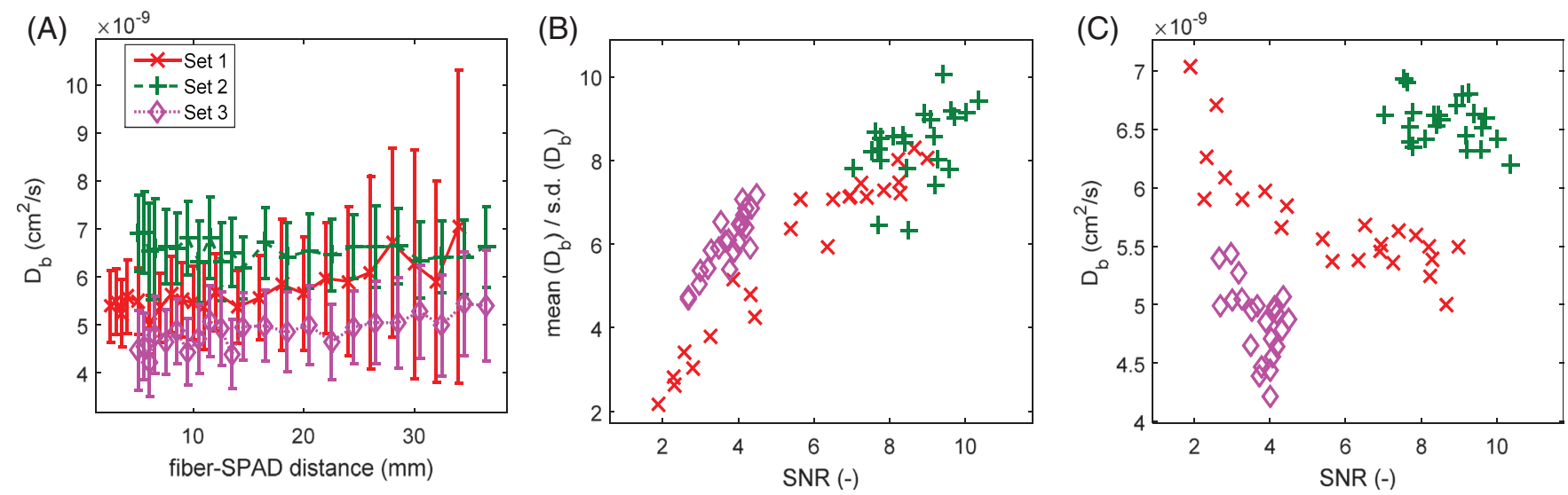

F I G URE 8 (A) Fitted $D_{\mathrm{b}}$ when changing fiber-SPAD separation. Error bars correspond to mean and SD over 100 sets of $16.4 \mathrm{~ms}$ measurements. (B) The stability of the $D_{\mathrm{b}}$ estimates tended to increase with increasing signal-to-noise ratio (SNR). This increase was statistically significant at $P<.001$ for linear regressions of Sets 1 and 3 and at $P<.05$ for Set 2. (C) There is also a tendency for the $D_{\mathrm{b}}$ values themselves to increase with decreased SNR. This increase was statistically significant at $P<.001$ for Set 1 , not significant for Set 2 and statistically significant at $P<.02$ for Set 3

(A)



(B)



(C)

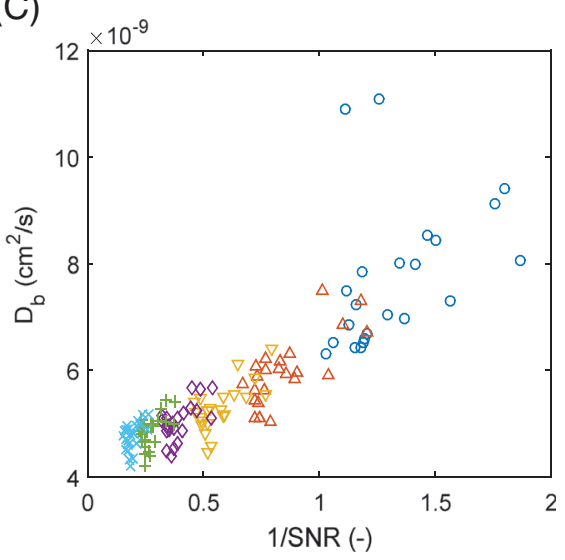

F I G URE 9 (A) Impact of the contiguous repetition length on signal-to-noise ratio (SNR). The square root of the repetition length normalized with the SNR for $g_{2}$ at the longest repetition length is plotted for reference. The SNR decreases somewhat faster than the square root of the repetition length as $1 \mu \mathrm{s}$ to $1 \mathrm{~ms}$ of the signal must be reserved for the delay time, $\tau$. The error bars corresponds to mean and SD over all fiber-detector distances. (B) Mean $D_{\mathrm{b}}$ over the SD of $D_{\mathrm{b}}$, that is, the SNR of the $D_{\mathrm{b}}$ estimates, increases linearly with the SNR of $g_{2}(P<.001)$. (C) Mean $D_{\mathrm{b}}$ estimates increase as the SNR of $g_{2}$ decreases $(P<.001)$ with $D_{\mathrm{b}}$ approximately linearly proportional to $1 / \mathrm{SNR}$. The intercept at 0 gives an extrapolated $D_{\mathrm{b}}$ estimate of $4.0 \times 10^{-9} \mathrm{~cm}^{2} / \mathrm{s}$ for a perfect signal with infinite SNR here when doing linear regression between $D_{\mathrm{b}}$ and $1 / \mathrm{SNR}$

Examples of fits to $g_{2}$ and the $D_{\mathrm{b}}$ values when changing temperature are presented in Figure 10 . The $D_{\mathrm{b}}$ values increased approximately linearly with temperature as expected from Equation (9) due to the approximately linear decrease in the inverse of the viscosity [34].

The blood occlusion test is presented in Figure 11. The fitted $D_{\mathrm{b}}$ was greatly reduced from $2.5 \times 10^{-9} \pm 0.6 \times$ $10^{-9} \mathrm{~cm}^{2} / \mathrm{s}$ during baseline to $0.4 \times 10^{-9} \pm 0.2 \times$ $10^{-9} \mathrm{~cm}^{2} / \mathrm{s}$ during occlusion. This was followed by a peak of $11.7 \times 10^{-9} \mathrm{~cm}^{2} / \mathrm{s}$ in the beginning of the reperfusion.

Results for the different source-detector fiber separations in the Intralipid phantoms are presented in Figure 12. The fitted $D_{\mathrm{b}}$ went from $12.8 \times 10^{-9} \pm 0.9 \times 10^{-9} \mathrm{~cm}^{2} / \mathrm{s}$ at
$6 \mathrm{~mm}$ source-detector fiber separation to $27.6 \times 10^{-9} \pm 21.6$ $\times 10^{-9} \mathrm{~cm}^{2} / \mathrm{s}$ at $25 \mathrm{~mm}$ source-detector fiber separation. The increase in $D_{\mathrm{b}}$ estimate and variability was particularly pronounced at the two largest separations above $14 \mathrm{~mm}$. SNR at $2 \mu$ s decreased from 22.2 for the $6 \mathrm{~mm}$ separation to 1.4 for the $25 \mathrm{~mm}$ separation.

\section{4 | DISCUSSION}

In this study, a multipixel DCS system for blood flow measurements has been created and tested on milk phantoms and in a blood occlusion test in vivo. In a clinical setting, the 
FIGURE $10 \quad D_{\mathrm{b}}$ from changing temperature with measurements using the $200 \mu \mathrm{m}$ fiber at $4.6 \mathrm{~mm}$ sourcedetector fiber separation.

(A) Examples of fits to $g_{2}$ at a measurement time of $16.4 \mathrm{~ms}(2.5 \mathrm{~mm}$ fiber-SPAD distance). (B) Mean and SD over 100 repetitions for fits to $g_{2}$ at different temperatures and fiber-SPAD distances

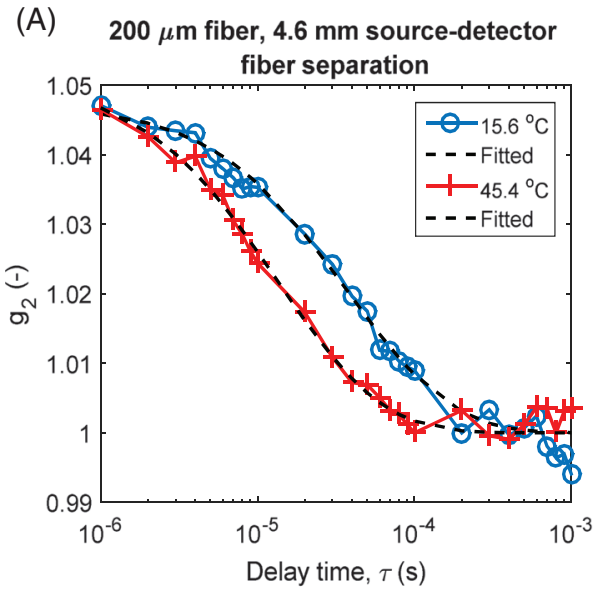

(B) $200 \mu \mathrm{m}$ fiber, $4.6 \mathrm{~mm}$ source-detector

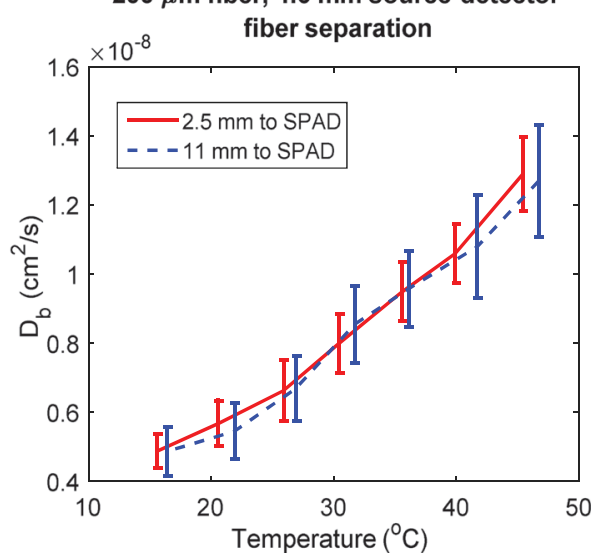



F I G U R E $11 D_{\mathrm{b}}$ measured on the volar forearm during the blood occlusion test using a source-detector fiber separation of $5 \mathrm{~mm}$ and a fiberSPAD distance of $11 \mathrm{~mm}$ (mean values over 10 repetitions in each time point). After 5 minutes of baseline measurement, the blood flow is occluded with a pressure cuff, greatly reducing $D_{\mathrm{b}}$. The cuff is released after a further 5 minutes and a typical increase in $D_{\mathrm{b}}$ can be seen during the reperfusion of the arm. It should be noted that blood flow is not actually Brownian in nature and the term $6 \alpha D_{\mathrm{b}}$ is usually called a blood flow index instead for in vivo measurements like this
Brownian diffusion coefficient, $D_{\mathrm{b}}$, is representing a relative blood flow index in the tissue and this has been found to provide better fitting than random flow models that could otherwise have been expected to fit better for blood vessels [5]. In the milk phantoms, on the other hand, it is actual Brownian diffusion.

The multipixel approach is beneficial by allowing improved SNR from independent speckle areas from the same collected light cone compared to just using 1 pixel in our system. The SNR benefitted from more speckle areas in each individual pixel too. It may be surprising that good autocorrelation signals are obtained with multimode fibers as very thin single-mode fibers usually are used for DCS. For example, He et al tried to collect light with a 1-mm diameter multimode fiber for DCS and got a flat line in $g_{2}$ while they got improved SNR with a few-mode detector fiber compared to a single-mode fiber [16]. However, as each detector pixel in our system is so small, only $50 \times$ $50 \mu \mathrm{m}^{2}$, only a small fraction of the light cone will hit it and the number of speckles on each will be limited even though a relatively large detector fiber is used. For comparison, Excelitas SPCM-AQRH SPADs typically used for DCS [35] have a diameter of $180 \mu \mathrm{m}$, giving a 10 times larger detector area than the detector pixels used by us. As can be seen in
F I G URE 12 (A) $D_{\mathrm{b}}$ estimates in $4 \%$ Intralipid phantom ( $0.8 \%$ lipid) at source-detector fiber separations of 6 to $25 \mathrm{~mm}$. Mean and SD over 100 repetitions at a measurement time of $32.8 \mathrm{~ms}$. (B) SNR vs $\tau$ for the same measurements. Mean SNR between 1 and $100 \mu$ s was 0.55 for the $25 \mathrm{~mm}$ separation $(\mathrm{SNR}=1.4$ at $2 \mu \mathrm{s})$

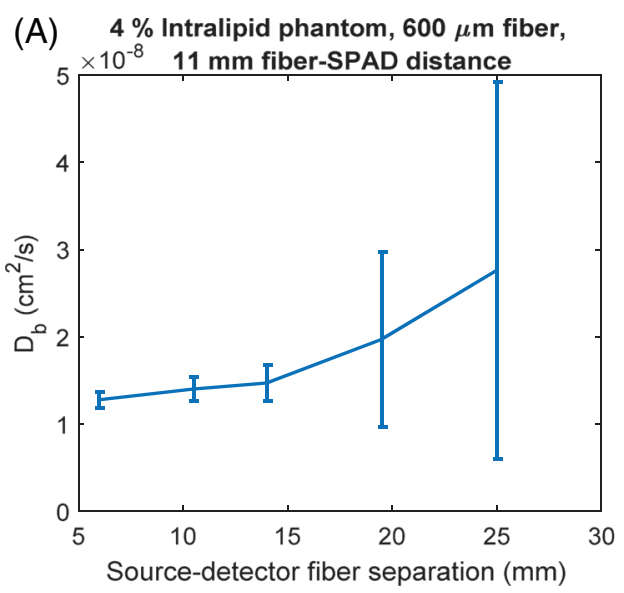

(B)

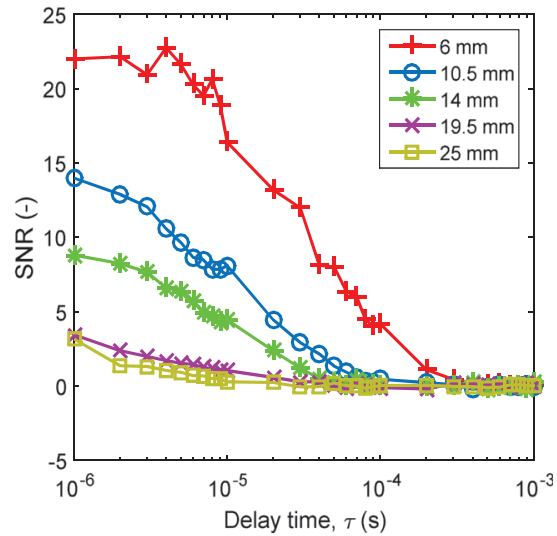


Figure 5, the signal quality of $g_{2}$ does indeed start to degrade when the larger fiber is moved closest to the SPAD and it would be expected that it would degrade further when moving closer. Early DCS literature justified the need of single-mode detector fibers by assuming speckle areas of approximately $1 \mu \mathrm{m}^{2}$ from multiple scattered light [36]. The $600 \mu \mathrm{m}$ diameter detector fiber would actually need to be as close as $0.7 \mathrm{~mm}$ to the SPAD in our system in order to obtain such small speckle areas according to Equation (2). Zhou et al [22] also obtained good signal quality by the use of a multimode detector fiber over a 512-pixel line-scan CMOS camera in the similar technique interferometric diffusing wave spectroscopy. They used a considerably stronger laser of $50 \mathrm{~mW}$ and a more complicate setup with fiber couplers, attenuator and beam splitter for mixing of additional unscattered source light with the scattered light on the detector. At present, the cost of CMOS cameras with thousands of pixels and SPAD sensors with few tens of pixels are similar in the order of a few thousand euros, mainly because SPADs are produced in low quantities for research purposes only. It is expected that the cost of SPAD detectors will decrease in future years, as consumer electronics industry is starting to demonstrate interest for such technology in, for example, smartphones and automotive for LiDAR applications.

A pair of interesting effects probably related to the SPAD itself can be seen for the highest and lowest intensities (Figure 6). Lower values of $g_{2}(1 \mu \mathrm{s})$ than the fitted curve and even than for $g_{2}(2 \mu \mathrm{s})$ could be seen for the highest intensities (Figure 6B). This is likely an artifact of the hold-off time eliminating photon counts in the subsequent time bin as a new photon cannot be detected within this time frame (dead time). The risk of the hold-off time eliminating a subsequent photon is higher when the photon count rate is higher. For the lowest intensities on the other hand, $g_{2}(1 \mu \mathrm{s})$ tended to be higher than the fitted curve. This is likely due to afterpulsing generating false photon counts in the subsequent time bin, adding false extra correlation for the shortest delay time. This will have a relatively higher impact when the photon count rate is low. The effects are in accordance to what have been predicted for DCS in computer simulations [37]. Afterpulsing and hold-off time are connected as setting a shorter hold-off time increases the risk of afterpulsing. Something to note here is that the shortest delay time often is used to estimate $\beta$ in DCS. This might be suboptimal as this is the delay time most affected by hold-off time and afterpulsing.

Another interesting effect is that the $D_{\mathrm{b}}$ estimates themselves increase as the SNR decreases (Figure 9C), that is. a noisier signal may be misinterpreted as an increase in blood flow. This is the opposite effect compared to the related technique laser speckle imaging, where more noise will give more speckle contrast in the image and thus reduce the blood flow estimate unless the noise is estimated and compensated for
[4]. It is likely that similar noise compensation would be beneficial in DCS. In that case, the noise should probably not be estimated in vivo in the same manner as in this study as changes in blood flow over the heart cycle will introduce variability that is not noise but will lower the SNR estimate. Zhou et al [22] (Supplementary Material) stabilized $D_{\mathrm{b}}$ estimates for long source-detector fiber separations in diffusing wave spectroscopy by adding a noise term to the unnormalized electric field autocorrelation function, $G_{1}$ (Equation (3) without denominator).

The current system is a bit limited by the firmware which is only able to send contiguous signals that are relatively limited in time. Being able to send longer contiguous signals would improve the SNR by allowing to use more SPADs of the array to further improve the signal by averaging the autocorrelation functions from them. Using many pixels of a single detector array is a much more resource-efficient method than, for example, using multiple detector fibers to separate detectors. The latter has been a strategy used in other studies for signal improvement in DCS at long source-detector separations or to improve the temporal resolution of the $D_{\mathrm{b}}$ estimates $[17,38]$. It would also be beneficial to reduce the current delay of $42 \mathrm{~ms}$ between each measurement, both for faster measurements in general and in case a high temporal resolution to study the changes over the heart cycle is desired.

Currently, all analysis is done in postprocessing. This bypasses the need of expensive correlator boards typically used in DCS but does not allow for real-time monitoring of blood perfusion changes and generates much larger data files than if just the $g_{2}$ itself was recorded. These are in particularly drawbacks if the system is to be used for long-time monitoring. An elegant and cheap solution to this would be to calculate $g_{2}$ in the recording software. Such software solution for DCS has been implemented by Wang et al [18].

It could be considered to use a detector with enhanced sensitivity at $780 \mathrm{~nm}$ or a stronger laser in order to obtain more light when source-detector fiber separation is large. The Department of Biomedical Engineering at Linköping University has traditionally worked with LDF rather than DCS and only low power coherent lasers were thus available for this study. Using a stronger laser has the drawback of requiring more safety considerations regarding eye protection and avoidance of tissue heating when using the system clinically. He et al [16] tested phantoms with $\mu_{\mathrm{s}}^{\prime}=8 \mathrm{~cm}^{-1}$ and a source-detector fiber separation of $2.5 \mathrm{~cm}$. At $\tau=2 \mathrm{~ms}$, they obtained a peak SNR around 15 with 1 second acquisition time and a standard $100 \mathrm{~mW}$ DCS laser. In our study, an SNR of 1.4 was obtained at $\tau=$ $2 \mathrm{~ms}$ for the same source-detector separation in the Intralipid phantom with corresponding scattering and slightly lower absorption. An 100-fold increase in power to $60 \mathrm{~mW}$ should be expected to give an SNR improvement of $\sqrt{100}=10$ and thus result in similar SNR at a much lower acquisition time. 
Increasing detector sensitivity and using more detector pixels should improve the SNR further. With a sufficiently large number of detector pixels, large separation measurements should be possible to make also with the current power of $0.6 \mathrm{~mW}$. This has the advantage of a cheaper, more portable laser that is safer for the eyes.

Active pixel sensors (APSs) have detection efficiency in the near infrared typically higher than SPAD sensors (about $50 \%$ for APS vs $15 \%$ for SPADs [39]). Nevertheless, the advantage of a SPAD camera is the single photon sensitivity that allowed us to work with very short integration times $(1 \mu \mathrm{s})$ and low count rate per frame (less than 10 photons). This would not be possible with a CMOS camera, which has no negligible readout noise [39].

A laser with a somewhat shorter wavelength (to match the peak efficiency of silicon detectors) could also be considered but is limited by the rapidly increasing tissue absorption beyond the optical window of 650 to $950 \mathrm{~nm}$. Further, the used wavelength of $780 \mathrm{~nm}$ has the benefit of being close to the isosbestic point around $800 \mathrm{~nm}$ where $\mu_{\mathrm{a}}$ of oxygenated and deoxygenated blood are equal [40, 41]. Impact of changes in $\mu_{\mathrm{a}}$ from changes in blood oxygenation on the perfusion estimates is thus limited. An increase in $\mu_{\mathrm{a}}$ which is not accounted for the fitting should shift $g_{2}$ rightwards to longer delay times and thus give lower $D_{\mathrm{b}}$ estimates [42]. However, an increase in $\mu_{\mathrm{a}}$ will also lower the detected light intensity. As can be seen in Figure 9C, this could increase the $D_{\mathrm{b}}$ estimate. The relation is thus not trivial.

A benefit of the SPAD used in this study is that it is not vulnerable to high light intensities and will provide reliable results unless being too close to the limitation of bit rate or nearing count rate saturation at 1 per hold-off time, which in this case of using a hold-off time of 50 ns would correspond to a count rate of $20 \mathrm{Mcps}$. Other avalanche detectors may have a very nonlinear response if the photon count is too high or even take damage.

\section{5 | CONCLUSION}

In conclusion, the prototype system works well, obtaining improved SNR when averaging $g_{2}$ from multiple pixels and, for a limited range, when collecting multiple speckles per pixel. Hardware improvements and further studies regarding, for example, the data acquisition and optimal settings will further improve the achievable results.

\section{OR CID}

Johannes D. Johansson (10 https://orcid.org/0000-00034910-0291

\section{REFERENCES}

[1] I. Fredriksson, C. Fors, J. Johansson, Laser Doppler flowmetrya theoretical framework, http://insidan.imt.liu.se/bit/ldf/ldfmain. html 2007 (accessed: January 1, 2018).

[2] G. Nilsson, G. Salerud, T. Strömberg, K. Wårdell, M. Larsson, in Biomedical Photonics Handbook (Ed: T. Vo-Dinh), CRC Press, Boca Raton, FL 2003, p. 15:11.

[3] T. Dragojevic, D. Bronzi, H. M. Varma, C. P. Valdes, C. Castellvi, F. Villa, A. Tosi, C. Justicia, F. Zappa, T. Durduran, Biomed. Opt. Express 2015, 6, 2865.

[4] D. A. Boas, A. K. Dunn, J. Biomed. Opt. 2010, 15, 011109.

[5] T. Durduran, R. Choe, W. B. Baker, A. G. Yodh, Rep. Prog. Phys. 2010, 73, 076701.

[6] J. D. Johansson, M. Mireles, J. Morales-Dalmau, P. Farzam, M. Martinez-Lozano, O. Casanovas, T. Durduran, Biomed. Opt. Express 2016, 7, 481.

[7] M. G. D. Karlsson, C. Fors, K. Wårdell, H. Casimir-Ahn, Med. Biol. Eng. Comput. 2005, 43, 582.

[8] S. A. Pape, R. D. Baker, D. Wilson, H. Hoeksema, J. C. Jeng, R. J. Spence, S. Monstrey, Burns 2012, 38, 187.

[9] H. Jonasson, S. Bergstrand, F. H. Nyström, T. Länne, C. J. Östgren, N. Bjarnegård, I. Fredriksson, M. Larsson, T. Strömberg, Diab. Vasc. Dis. Res. 2017, 14, 363.

[10] P. Zsigmond, S. Hemm-Ode, K. Wårdell, Stereotact. Funct. Neurosurg. 2018, 95, 392.

[11] M. Stucker, M. Esser, M. Hoffmann, U. Memmel, A. Hirschmuller, C. von Bormann, K. Hoffmann, P. Altmeyer, Acta Derm. Venereol. 2002, 82, 25.

[12] H. Jonasson, I. Fredriksson, A. Pettersson, M. Larsson, T. Strömberg, Microvasc. Res. 2015, 102, 70.

[13] H. S. Yazdi, T. D. O'Sullivan, A. Leproux, B. Hill, A. Durkin, S. Telep, J. Lam, S. S. Yazdi, A. M. Police, R. M. Carroll, F. J. Combs, T. Strömberg, A. G. Yodh, B. J. Tromberg, J. Biomed. Opt. 2017, 22, 45003.

[14] P. Farzam, J. Johansson, M. Mireles, G. Jimenez-Valerio, M. Martinez-Lozano, R. Choe, O. Casanovas, T. Durduran, Biomed. Opt. Express 2017, 8, 2563.

[15] E. M. Buckley, N. M. Cook, T. Durduran, M. N. Kim, C. Zhou, R. Choe, G. Yu, S. Schultz, C. M. Sehgal, D. J. Licht, P. H. Arger, M. E. Putt, H. H. Hurt, A. G. Yodh, Opt. Express 2009, 17, 12571

[16] L. He, Y. Lin, Y. Shang, B. J. Shelton, G. Q. Yu, J. Biomed. Opt. 2013, 18, 037001 .

[17] G. Dietsche, M. Ninck, C. Ortolf, J. Li, F. Jaillon, T. Gisler, Appl. Optics 2007, 46, 8506.

[18] D. T. Wang, A. B. Parthasarathy, W. B. Baker, K. Gannon, V. Kavuri, T. Ko, S. Schenkel, Z. Li, Z. R. Li, M. T. Mullen, J. A. Detre, A. G. Yodh, Biomed. Opt. Express 2016, 7, 776.

[19] L. Gagnon, M. Desjardins, J. Jehanne-Lacasse, L. Bherer, F. Lesage, Opt. Express 2008, 16, 15514.

[20] K. Verdecchia, M. Diop, A. Lee, L. B. Morrison, T. Y. Lee, K. St Lawrence, Biomed. Opt. Express 2016, 7, 3659.

[21] M. Pagliazzi, S. K. V. Sekar, L. Colombo, E. Martinenghi, J. Minnema, R. Erdmann, D. Contini, A. Dalla Mora, A. Torricelli, A. Pifferi, T. Durduran, Biomed. Opt. Express 2017, 8, 5311.

[22] W. J. Zhou, O. Kholiqov, S. P. Chong, V. J. Srinivasan, Optica 2018, 5,518 . 
[23] F. Zappa, S. Tisa, A. Tosi, S. Cova, Sensor Actuat. A 2007, 140, 103.

[24] F. Villa, D. Bronzi, Y. Zou, C. Scarcella, G. Boso, S. Tisa, A. Tosi, F. Zappa, D. Durini, S. Weyers, U. Paschen, W. Brockherde, J. Mod. Optic. 2014, 61, 102.

[25] A. E. Siegman, Proc. IEEE 1966, 54, 1350.

[26] C. Zhou, G. Q. Yu, D. Furuya, J. H. Greenberg, A. G. Yodh, T. Durduran, Opt. Express 2006, 14, 1125.

[27] L. H. Kou, D. Labrie, P. Chylek, Appl. Optics 1993, 32, 3531.

[28] T. Lindbergh, I. Fredriksson, M. Larsson, T. Strömberg, Opt. Express 2009, 17, 1610.

[29] A. Einstein, Ann. Phys. 1905, 322, 549.

[30] E. W. Lemmon, A. H. Harvey, in CRC Handbook of Chemistry and Physics (Ed: J. R. Rumble). CRC Press, Boca Raton, Fl 2017.

[31] S. H. Tseng, A. Grant, A. J. Durkin, J. Biomed. Opt. 2008, 13, 014016.

[32] P. X. Lai, X. Xu, L. H. V. Wang, J. Biomed. Opt. 2014, 19, 035002 .

[33] H. Assadi, R. Karshafian, A. Douplik, Int. J. Photoenergy 2014, 1.

[34] J. D. Johansson, PhD Thesis, Linköping University, Linköping, Sweden 2008.
[35] T. Durduran, A. G. Yodh, Neuroimage 2014, 85, 51.

[36] D. A. Boas, A. G. Yodh, J. Opt. Soc. Am. A 1997, 14, 192.

[37] P. R. Donoso, Master Thesis, Universitat Politècnica de Catalunya (Barcelona, Spain) 2015.

[38] E. M. Buckley, A. B. Parthasarathy, P. E. Grant, A. G. Yodh, M. A. Franceschini, Neurophotonics 2014, 1.

[39] D. Bronzi, F. Villa, S. Tisa, A. Tosi, F. Zappa, IEEE Sens. J. 2016, 16, 3 .

[40] S. Takatani, M. D. Graham, I.E.E.E. Trans. Biomed. Eng. 1979, 26, 656.

[41] S. Prahl, Optical absorption of hemoglobin, https://omlc.org/ spectra/hemoglobin/ 1999 (accessed: January 2018).

[42] D. Irwin, L. X. Dong, Y. Shang, R. Cheng, M. Kudrimoti, S. D. Stevens, G. Q. Yu, Biomed. Opt. Express 2011, 2, 1969. 